\title{
Simplified Modeling of Ring Resonators and Split Ring Resonators Using Magnetization
}

\author{
Dongho Jeon $\cdot$ Bomson Lee*
}

\begin{abstract}
This paper examines various aspects of the electromagnetic responses of the ring resonator located in the transverse electromagnetic cell. In addition, an equivalent circuit for the ring resonator is proposed and analyzed based on the electromagnetic phenomenon of the resonator. The equivalent circuit was simply modeled based on the concept of magnetization. A method for achieving a wider operating bandwidth of the negative permeability is provided. The ring resonator with its resonant frequency of $13.56 \mathrm{MHz}$ was designed and its characteristics were examined in terms of S-parameters, effective permeability, loss rate, bandwidth, etc. The circuit and electromagnetic simulation results show an excellent agreement as well as that of theory.
\end{abstract}

Key Words: Metamaterial, Split Ring Resonator, Negative Permeability.

\section{Introduction}

The realization of media having negative permittivity and permeability, so called left-handed metamaterial, became feasible after 1991 when Pendry et al. [1] proposed a new method that used an array of thin wires and split ring resonators (SRRs). A number of approaches followed in many aspects in an effort to realize similar left-handed characteristics [2-5]. However, most of these showed high losses and narrow operating bandwidth (having a very sharp slope near the resonant frequency). In fact, the problem of SRRs has seldom been approached seriously from an engineering viewpoint, although many trials have been made. Basically, SRRs are similar to the ring resonator in their characteristics. In this paper, we model the ring resonator (SRRs) starting from the definition of a magnetic dipole moment and leading to a useful equivalent circuit. The mechanism of SRRs is explained with more familiar terms than in [1]. In addition, we provide a method of realizing the negative permeability over a large bandwidth. It is believed that the presented modeling will provide significant convenience and flexibility for the realization of the left-handed materials.

\section{II . Novel Modeling of a Ring Resonator (SRRs)}

We have modeled the ring resonator using an equivalent circuit. The dimensions of the ring resonator and its orientation with respect to the given transverse electromagnetic (TEM) wave are depicted in Fig. 1. The wave travels in the $\mathrm{z}$-direction with the electric and magnetic fields oriented in the $\mathrm{x}$ - and $\mathrm{y}$-directions, respectively. The radius of the loop is $r$ and the radius of the ring is $r_{\text {ring. }}$. The side length of the unit cell is $a$. $C$ is the value of the chip capacitor. It is inserted for resonance of the ring resonator which has some inductance $L$ originally. The total resistance $R$ of the ring resonator is given by

$$
R=R_{r}+R_{l}+R_{L}
$$

where $R_{r}$ is the radiation resistance, $R_{l}$ is the ohmic resistance, and $R_{L}$ is an additionally loaded resistance which is 0 originally, but can be added to help the resonator to resonate weakly. When $R_{L} \rightarrow \infty$, the current cannot flow on the resonator and the resonator becomes inactive. As $R_{L}$ is varied, the $Q$ value of the resonator is changed and a wider operating bandwidth of the negative permeability may be achieved. As the magnetic field $H_{0}$ is passes through the ring resonator, a voltage $V_{\text {emf }}$ will be induced as shown in Eq. (2) based on Faraday's law.

Manuscript received January 28, 2013 ; Revised February 27, 2013 ; Accepted April 2, 2013. (ID No. 20130128-003J)

Department of Electronics and Radio Engineering, College of Electronics and Information, Kyung Hee University, Yongin, Korea.

${ }^{*}$ Corresponding Author : Bomson Lee (e-mail : bomson@khu.ac.kr)

This is an Open-Access article distributed under the terms of the Creative Commons Attribution Non-Commercial License (http://creativecommons.org/licenses/ by-nc/3.0) which permits unrestricted non-commercial use, distribution, and reproduction in any medium, provided the original work is properly cited. 


$$
v_{\text {emf }}=\frac{\partial\left(\mu_{0} H_{0} \pi r^{2}\right)}{\partial t} \stackrel{\frac{\partial}{\partial t}=j \omega}{\longrightarrow} V_{e m f}=j \omega \mu_{0} H_{0} \pi r^{2}
$$

Accordingly, the current $I$ can be easily determined as Eq. (3) simply by dividing $V_{\text {emf }}$ with the resonator impedance $Z(\omega)$ (series sum of $R, L$, and $C$ ).

$$
I(\omega)=\frac{V_{\text {emf }}}{Z(\omega)}=\frac{j \omega \mu_{0} H_{0} \pi r^{2}}{R+j R Q\left(\frac{\omega}{\omega_{0}}-\frac{\omega_{0}}{\omega}\right)}[A]
$$

The magnetization $M$ and the relative effective permeability can be obtained as (4) and (5), respectively.

$$
\begin{aligned}
& \bar{M}(\omega)=\frac{\bar{m}(\omega)}{\Delta v}=-\frac{j \omega \mu_{0} H_{0}\left(\pi r^{2}\right)^{2}}{\left[R+j R Q\left(\frac{\omega}{\omega_{0}}-\frac{\omega_{0}}{\omega}\right)\right] a^{3}} \overline{a_{y}}=\chi_{m}(\omega) H_{0} \bar{a}_{y} \\
& \mu_{e f f}(\omega)=1+\chi_{m}(\omega)=1-\frac{j \omega \mu_{0}\left(\pi r^{2}\right)^{2}}{a^{3}\left[R+j R Q\left(\frac{\omega}{\omega_{0}}-\frac{\omega_{0}}{\omega}\right)\right]}
\end{aligned}
$$

where $\omega$ is angular frequency, $\omega_{0}$ is the resonant frequency, $\bar{m}(\omega)$ is magnetic dipole moment $\left(\bar{m}=I(\omega) \pi r^{2} \overline{a_{y}}\right)$, $Q$ is the value of $Q$ factor written as Eq. (6), $\chi_{m}$ is magnetic susceptibility, and $\mu_{0}$ is the permeability in free space $\left(\mu_{0}=4 \pi \times 10^{-7}[\mathrm{H} / \mathrm{m}]\right)$.

$$
Q=\frac{\omega_{0} L}{R}
$$

The parameter $L$ in Eq. (6) indicates the self-inductance of the proposed ring resonator. Now, we want to devise an equivalent circuit for the structure in Fig. 1. By multiplying the derived relative effective permeability with the geometrical factor $(g)$ which is determined for the cross-sectional shape of a specific transmission line, we can obtain the effective inductance of the transmission line.

$$
L_{e f f}(\omega)=\mu_{0} \mu_{e f f}(\omega) g[H / m]
$$

For an instance, $g$ for the parallel plate waveguide transmission line with width $W$ and height $h$ is given by $h / W$. Now, the series impedance of the unit cell including the ring resonator can be expressed as

$$
\begin{aligned}
& Z(\omega)=j \omega L_{\text {eff }}(\omega) d \\
& =j \omega \mu_{0} g d+\frac{1}{\frac{a^{3}}{\left(\omega \mu_{0} \pi r^{2}\right)^{2} g d}\left[R+j \omega_{0} L\left(\frac{\omega}{\omega_{0}}-\frac{\omega_{0}}{\omega}\right)\right]^{[\Omega]}}
\end{aligned}
$$

where $d$ is the physical length of the transmission line unit cell. A close examination of (8) leads to the equi- valent circuit depicted in Fig. 2. The equivalent circuit consists of a right handed $(\mathrm{RH})$ transmission line with $L_{e q} \cdot d, C_{e q} \cdot d$ and a parallel $G^{\prime}, C^{\prime}, L^{\prime}$ resonator in the series branch. The $\mathrm{RH}$ transmission line may be alternatively represented by $Z_{\mathrm{c}}$ and $k d$. The total series impedance of the equivalent circuit in Fig. 2 can be written as

$$
Z_{e q}(\omega)=j \omega L_{e q} d+\frac{1}{G^{\prime}+j \omega_{0} C^{\prime}\left(\frac{\omega}{\omega_{0}}-\frac{\omega_{0}}{\omega}\right)}[\Omega]
$$

The value of parameters $G^{\prime}, C^{\prime}, L^{\prime}$ in Eq. (9) can be obtained as Eq. (10) by comparing the Eqs. (8) and (9).

$$
G^{\prime}=\frac{a^{3}}{\left(\omega \mu_{0} \pi r^{2}\right) g d} R, C^{\prime}=\frac{a^{3}}{\left(\omega \mu_{0} \pi r^{2}\right) g d} L, L^{\prime}=\frac{1}{\omega_{0}^{2} C}
$$

\section{Simulation Results and Discussion}

The ring resonator used in the electromagnetic (EM) simulation is made of copper and is designed at 13.56 MHz. The unit cell size $(a)$ is $12 \mathrm{~cm}\left(0.005 \lambda_{0}\right)$. The radius of the loop $(r)$ and the ring $\left(r_{\text {ring }}\right)$ are $5 \mathrm{~cm}$ and 1 $\mathrm{mm}$, respectively. The ohmic resistance $R_{l}$ is $0.058 \Omega$ and the radiation loss $R_{r}$ is negligible $\left(R_{L}=0 \quad \Omega\right.$ assumed). The value of capacitance for the resonance is 533 pF. Fig. 3(a) and (b) show the circuit (based on Fig. 2) and EM-simulated magnitude and phase of the S-parameters for the ring resonator when the TEM wave propagates in the z-direction as depicted in Fig. 1. The circuit based on Fig. 2 and the EM-simulated results show excellent agreement. The extracted real and imaginary parts of the effective permeability based on the S-parameter [6] and theory (4) are shown in Fig. 4(a) and (b), respectively. The excellent agreement validates this modeling. In Fig. 5(a), the real part of the effective permeability is shown with respect to the $Q$ values which can be controlled by varying the extra resistance $R_{L}$. For this case, the ring resonators have been designed to have the specific value for effective permeability $\left(\mu_{\text {eff }}=-1\right)$ of $13.56 \mathrm{MHz}$ (each case has different resonant frequencies).

Fig. 5(b) shows the loss rate of the ring resonator with different values of $Q$. The loss rate is defined as Eq. (11).

$$
\eta_{L}=1-\left|S_{11}\right|^{2}-\left|S_{21}\right|^{2}
$$

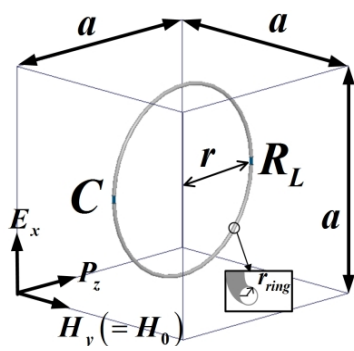

Fig. 1. Structure of a ring resonator. 


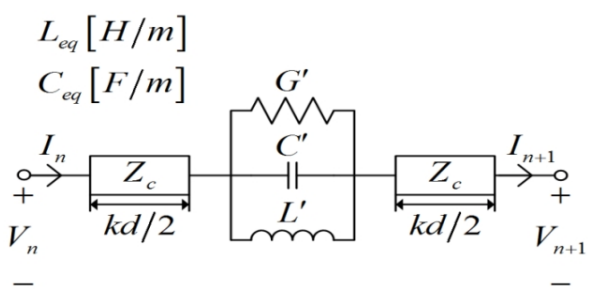

Fig. 2. Equivalent circuit of ring resonator.

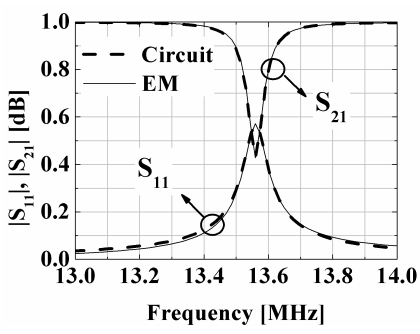

(a)

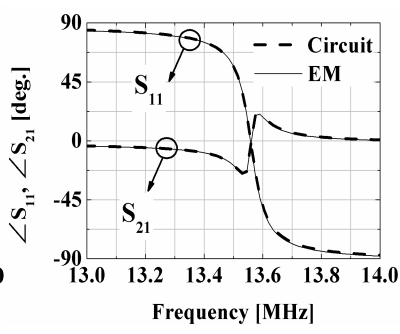

(b)
Fig. 3. Simulated $S$-parameters: (a) magnitude, (b) phase. EM $=$ electromagnetic.

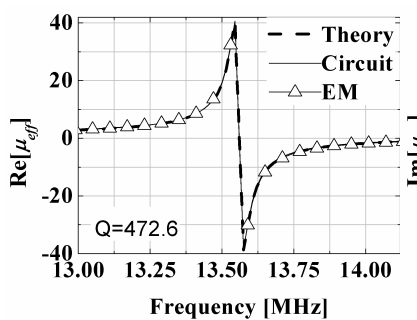

(a)

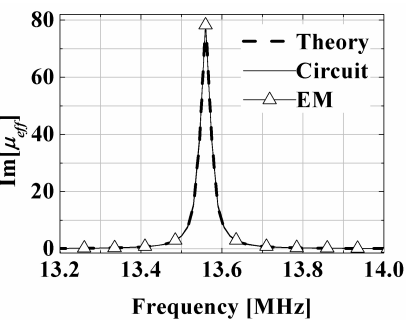

(b)
Fig. 4. Extracted effective permeability at $13.56 \mathrm{MHz}$ : (a) real, (b) imaginary. EM=electromagnetic.

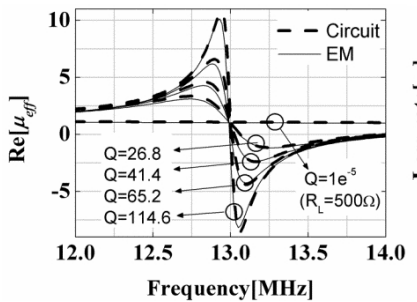

(a)

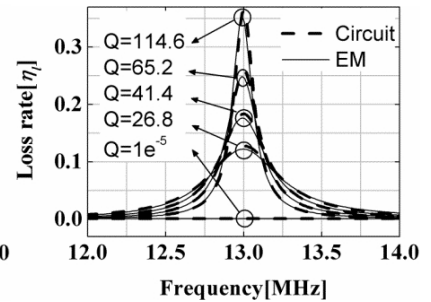

(b)
Fig. 5. Circuit/electromagnetic (EM)-simulated $\mu_{\text {eff }}$ and $\eta_{l}$ with respect to $R_{L}$. (a) $\operatorname{Re}\left[\mu_{\text {eff }}\right]$ (varing $R_{L}$ from 0.15 to $500 \Omega$ ), (b) Loss rate, $\eta_{l}$ (varing $R_{L}$ from 0.15 to $500 \Omega$ ).

Each case shows that the highest loss rate is shown at the resonant frequency, but loss rates are very low at the operating frequency of $13.56 \mathrm{MHz}\left(\eta_{l}\right.$ is less than $2.5 \%$ for each case).

Comparison of Figs. 4(a) and 5(a) shows that the resonance occurs less sensitively as the value of $Q$ is lowered by inserting extra resistance $R_{L}$ 's. Furthermore, the widest operating bandwidth of the effective permeability is achieved when the resonator has the specific value of
$Q\left(Q=26.8, R_{L}=0.8 \Omega\right)$. The widest bandwidth is only effective when the effective permeability is designed to have the value of -1 . The circuit and EM-simulated results are again in excellent agreement.

\section{Conclusion}

The effective permeability of a ring resonator (or SRR) has been formulated based on the concept of magnetization. Its equivalent circuit has also been proposed and analyzed with necessary comparisons. The circuit and EM simulated results are in excellent agreement. The drawback of the narrow-banded SRRs can be significantly ameliorated based on the proposed modeling and formulations. With this modeling, the problem of synthesizing the effective medium can be engineered more systematically.

This work was supported by Mid-career Researcher Program through the National Research Foundation of Korea (NRF) grant funded by the Korea government (No. 2012047938).

\section{References}

[1] J. B. Pendry, A. J. Holden, D. J. Robbins, and W. J. Stewart, "Magnetism from conductors and enhanced nonlinear phenomena," IEEE Transactions on Microwave Theory and Techniques, vol. 47, no. 11, pp. 2075-2084, Nov. 1999.

[2] S. M. Rudolph, C. Pfeiffer, and A. Grbic, "Design and free-space measurements of broadband, low-loss negative-permeability and negative-Index media," $I E$ EE Transactions on Antennas and Propagation, vol. 59, no. 8, pp. 2989-2997, Aug. 2011.

[3] K. Zhang, Q. Wu, J. H. Fu, F. Y. Meng, and L. W. $\mathrm{Li}$, "Metamaterials with tunable negative permeability based on mie resonance," IEEE Transactions on Magnetics, vol. 48, no. 11, pp. 4289-4292, Nov. 2012.

[4] C. Kim and B. Lee, "Analysis of magnetically coupled wireless power transmission for maximum efficiency," Journal of Electromagnetic Engineering and Science, vol. 11, no. 3, pp. 156-160, Dec. 2011.

[5] H. Luo, X. Wang, Z. Liao, T. Wang, and R. Gong, "Experimental investigation of $\mathrm{R}(\omega), \mathrm{T}(\omega)$, and $\mathrm{L}$ $(\omega)$ for multi-layer SRRs and wires metamaterial," Journal of Electromagnetic Engineering and Science, vol. 10, no. 3, pp. 186-189, Sep. 2010.

[6] S. G. Mao, S. L. Chen, and C. W. Huang, "Effective electromagnetic parameters of novel distributed lefthanded microstrip lines," IEEE Transactions on Microwave Theory and Techniques, vol. 53, no. 4, pp. 1515-1521, Apr. 2005. 